\title{
Das Timing der Familiengründung und dessen Folgen für Familien
}

\section{The timing of family formation and its consequences for families}

\begin{abstract}
Zusammenfassung;
Ausgehend vom lückenhaften Forschungsstand zu den Folgen des Timing von Elternschaft für die Lebenslage, Lebensform, und Lebensführung von Müttern, Vätern und Kindern werden Hypothesen $\mathrm{zu}$ diesem Zusammenhang entwickelt und anschließend mit Daten des DJI-Surveys AID:A für die Familiengründungsphase überprüft. Wo möglich, werden die Befunde mit Daten aus dem Mikrozensus 2007 validiert. Der Analyse sind Überlegungen zur Definition von ,früher“ und „später“ Elternschaft vorgeschaltet. Die Untersuchung zeigt, dass zentrale Aspekte der sozialen Lage und der Institutionalisierungsgrad der Paarbeziehung mit dem biografischen Zeitpunkt der Familiengründung variieren. Das Zeitbudget für das Kind und die Zufriedenheit der Eltern werden durch den biografischen Zeitpunkt der Familiengründung aber kaum beeinflusst.
\end{abstract}

Schlagwörter: Familiengründung, Elternschaft, Zeitverwendung, Zufriedenheit

\begin{abstract}
:
Little is known about the consequences of the timing of family formation for the living conditions, the civil status, the conduct of life and the well-being of mothers, fathers and their children. This study is based on the DJI Survey AID:A, and the official statistics of the 2007 German Microcensus. The analysis includes considerations about the definition of "early" and "late" parenthood. Results: Living conditions, educational background, marital status and the stability of partnership vary by the timing of the first child. The timing of family formation hardly has an impact on the time that parents spend with their first child nor does it influence their contentment.
\end{abstract}

Key words: family formation, parenthood, time use, contentment 


\section{Einleitung}

Wie in den vergangenen Jahren ging auch 2009 die durchschnittliche Zahl der Geburten von jüngeren Frauen zurück, während die Zahl der Geburten bei Frauen über 33 Jahren zunahm (Statistisches Bundesamt 2010). ${ }^{1}$ Dieser langfristige Trend zum „Aufschub“2 der Erstelternschaft ist demografisch relevant, weil er einerseits zu einer permanenten Unterschätzung der Geburtenrate (Payk/Schmider 2009; Scharein 2010) ${ }^{3}$ führt, aber auch real die Chance von Paaren verringert, erste und weitere Kinder zu bekommen (Statistisches Bundesamt 2007: 30).

Mit welchen Folgen das Timing der Familiengründung für die Lebenslage, Lebensführung und Lebensform der Eltern und ihrer Kinder einhergeht, fand bisher nur unsystematisch Beachtung. Deshalb soll hier der Frage nachgegangen werden, wie und mit welchen Ressourcen Elternschaft gelebt wird, wenn sie vergleichsweise früh, dem Durchschnitt entsprechend oder erst relativ spät im Lebenslauf gestartet wird. Unser Ziel ist es, einen Vergleich der Lebenslage, der Lebensführung und des Wohlbefindens von Familien in Abhängigkeit vom biografischen Zeitpunkt der Familiengründung vorzulegen. Dabei beschränken wir unsere Betrachtung auf Erwachsene, deren erstes Kind das siebte Lebensjahr noch nicht erreicht hat. So lassen sich unsere Befunde einer erweiterten Familiengründungsphase zuordnen. Wir nutzen Daten des DJI-Surveys AID:A 2009 und validieren diese mit dem Mikrozensus 2007, dem jüngsten Mikrozensus, der derzeit allgemein zugänglich ist. Im Sample sind sowohl zusammenlebende als auch alleinerziehende Mütter und Väter. ${ }^{4}$

\section{Zur Definition ,früher“ und „später“ Elternschaft}

Die Studien, die es bisher zu früher und später Elternschaft gibt, arbeiten mit unterschiedlichen Altersgrenzen, dabei werden die Altersgrenzen für Mütter einer bestimmten Timing-Gruppe stets früher als die für Väter angesetzt (Block/Schmidt 2009; Heinzelmann 2003; Fthenakis 1999; Klindworth et al. 2004: 155; Marsiglo/Cohan 1997). Das Setzen von Altersgrenzen wird selten begründet. Dies soll hier aber mit dem Abwägen verschiedener Optionen geschehen.

Zum langfristigen Trend Engstler/Lüscher 1991

2 Wir setzen das Wort „Aufschub“ in Anführungsstrichen, denn das, was in der demografischen Forschung als (aktiver) „Aufschub“ (postponement) bezeichnet wird, wird subjektiv sehr unterschiedlich erlebt, unter Umständen auch als verzweifeltes Warten oder später Wunsch (Herlyn/Krüger 2003; Sardadvar 2010).

3 Allein die Verzögerung der Geburten im Lebenslauf hat einen negativen Einfluss auf die Zahl der Geburten pro Jahr, weil sich die Geburten - im Vergleich zu einer Phase, in der das Timing im individuellen Lebenslauf konstant bleibt - über einen längeren Zeitraum erstrecken („Tempoeffekt“", Scharein 2010).

4 Wir weisen die allerziehenden Mütter und Väter nirgends gesondert aus, weil deren Fallzahlen im AID:A-Survey in den Timing-Gruppen zu gering sind. 


\subsection{Medizinische Nomenklatur}

In der Gynäkologie wird jede Schwangerschaft nach dem 35. Lebensjahr als späte Schwangerschaft bezeichnet und galt bis in die 1980er Jahre hinein automatisch als „Risikoschwangerschaft". Noch heute wird allen Schwangeren ab diesem Lebensalter eine Fruchtwasseruntersuchung empfohlen, was diese Altersgrenze im öffentlichen Bewusstsein fortgesetzt markiert. Viele der Risiken, die früher mit Schwangerschaften über 35 Jahren einhergingen, sind heute medizinisch allerdings deutlich besser als noch in den 1980er Jahren beherrschbar (Engstler/Lüscher 1991; Herlyn/Krüger 2003: 18). Während die ehemals fixierte Altersgrenze für späte Mütter ins Wanken gerät, sind Altersgrenzen für späte Vaterschaft in der Medizin gar nicht etabliert, auch wenn medizinische Risiken für das Kind diskutiert werden.

Schwangerschaften von unter 18-Jährigen gelten als Risikoschwangerschaften. Frühe Vaterschaft ist medizinisch nicht definiert. Was die frühe Elternschaft generell betrifft, scheinen in Deutschland die sozialen Normen deutlich rigider als die medizinische Problematisierung. Eine Anleihe bei der Medizin hilft also nur begrenzt, Altersgrenzen für frühe und späte Elternschaft festzulegen.

\subsection{Soziale Normen}

Wenig explizit, aber deshalb umso wirksamer scheint in Deutschland eine soziale Norm selbstverständlich akzeptiert, nach der Schwangerschaften minderjähriger Frauen bzw. von Frauen unter 20 Jahren hoch problematisch sind. So hält sich zum Beispiel die Bundeszentrale für gesundheitliche Aufklärung zugute, dass die Zahl dieser Schwangerschaften in Deutschland im internationalen Vergleich niedrig ist, und verbucht dies als „Erfolg“ ihres breiten Präventionskonzeptes (Bundeszentralle für gesundheitliche Aufklärung 2009: 9). Damit kann die Bundeszentrale offenbar auf einen breiten gesellschaftlichen Konsens rekurrieren.

Eine Umfrage unter 18- bis 44-jährigen Frauen förderte wenig einheitliche Altersnormen zu Tage: „Das beste Alter (Anfangsalter) für Frauen, Kinder zu bekommen“, liegt für $31 \%$ der befragten Frauen zwischen 20 und 23 Jahren, für $46 \%$ zwischen 24 und 26 Jahren und für 10\% zwischen 27 bis 29 Jahren (Institut für Demoskopie Allensbach 2004: $19)^{5}$. An diesen Zahlen ist ein mehrheitliches Plädoyer von Frauen für einen Einstieg in die Familiengründung abzulesen, der deutlich vor dem Durchschnittsalter von Frauen bei der Geburt des ersten Kindes von 28,8 Jahren liegt, nämlich bei 24,1 (Statistisches Bundesamt 2010; Institut für Demoskopie Allensbach 2004: 19). Die vorherrschenden Altersnormen setzen sich also in der Praxis nicht durch.

Eine von einer deutlichen Mehrheit vertretene Norm scheint darin zu bestehen, dass erst die Ausbildung und die ersten Berufsjahre abzuschließen seien, bevor man Kinder in die Welt setzen sollte. Dies meinen $85 \%$ der 18 - bis 44 -jährigen Bevölkerung (Institut für Demoskopie Allensbach 2004: 25). Damit ist der normativ abgesicherte „richtige“ Zeitpunkt stark von Ausbildungs- und Arbeitsmarkteffekten abhängig. Dies bedeutet, dass

5 Die restlichen Prozentanteile verteilen sich auf Antwortkategorien, die weniger als $6 \%$ der Befragten auf sich vereinen. $6 \%$ der Befragten machen darüber hinaus keine Angabe. 
auch soziale Normen keine klaren Anhaltspunkte für die Festlegung von Altersgrenzen für frühe und späte Elternschaft liefern.

\subsection{Empirisch begründete Altersgrenzen}

Statt auf medizinische Nomenklaturen oder auf Altersnormen zu rekurrieren, wird in diesem Beitrag die empirisch ermittelbare Altersverteilung von Müttern und Vätern bei der Geburt ihres ersten Kindes zur Grundlage einer Definition gemacht. Dabei wird auf den Mikrozensus 2007 zurückgegriffen $^{6}$ : die frühesten 25 Prozent werden als „frühe“ und die spätesten $25 \%$ als „späte“ Mütter oder Väter bezeichnet. Damit sind im folgenden Beitrag ,frühe“ Mütter solche, die vor dem 25. Lebensjahr ihr erstes Kind bekommen haben, „späte“ Mütter solche, die über 32 Jahre alt waren, als sie ihr erstes Kind bekamen (vgl. Tabelle 1). Die Berechnung der Altersgrenzen erfolgt nach Geschlecht getrennt, weil die Altersverteilung deutlich mit dem Geschlecht der Eltern variiert, denn nach dieser Definition rechnen zu den frühen Vätern all jene Väter, die vor dem 29. Lebensjahr ihr erstes Kind bekommen haben. Zu den „späten“ Vätern zählen wir diejenigen, die über 35 Jahre alt waren, als sie zum ersten Mal Vater wurden (vgl. Tabelle 1).

Tabelle 1: Altersgrenzen für frühe und späte Mutter- und Vaterschaft

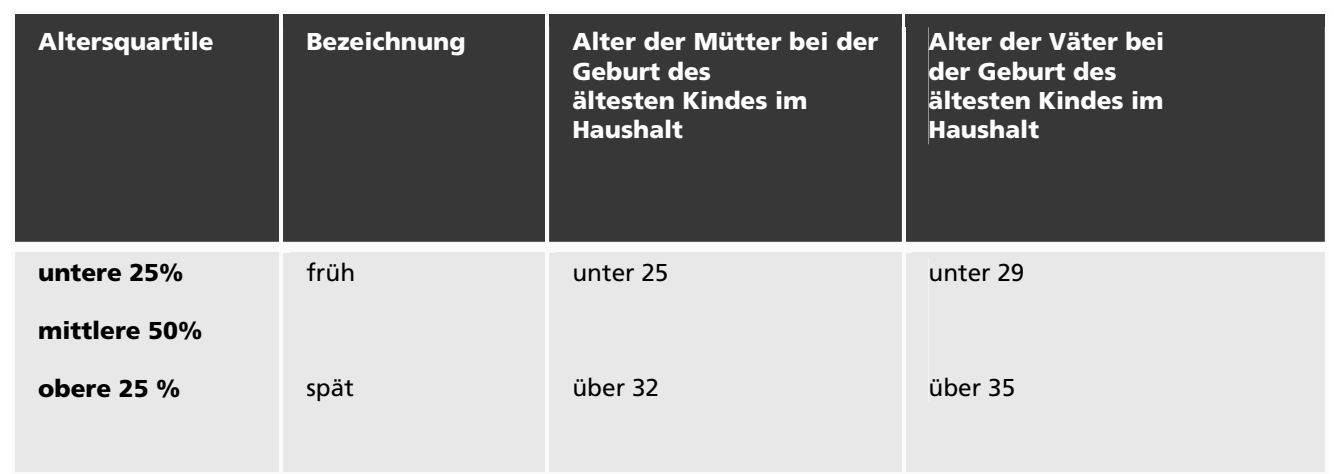

Basis Mikrozensus 2007: Frauen und Männer mit Kindern im Haushalt, die alle noch unter 7 Jahre alt sind. Obere und untere Altersquartile bzw. -dezile

Die in Tabelle 1 aufgeführten Altersgrenzen, die sich aus den Altersquartilen mit den Daten des Mikrozensus 2007 ergeben, werden im Abschnitt 5 genutzt, um die im Abschnitt 3 entwickelten Hypothesen zu überprüfen.

6 Da im Mikrozensus nicht die leiblichen Kinder, sondern die Kinder im Haushalt erhoben werden, weist Tabelle 1 nicht das Alter der Mütter und Väter bei der Geburt ihres ersten Kindes aus, sondern das Alter der Frauen und Männer bei der Geburt des ältesten Kindes im Haushalt. Diese Erwachsenen werden in Tabelle 1 als „Mütter“ und „Väter“ bezeichnet. 


\section{Theoretische Bezüge, Stand der Forschung und Hypothesen}

\subsection{Theoretische Bezüge}

Für die vorliegende Studie sind Lebenslage und Lebensführung zentrale theoretische Konzepte. Der DJI-Survey AID:A wurde auf der Basis des Lebenslagenansatzes entwickelt und erfasst neben der Höhe und Zusammensetzung des Haushaltseinkommens viele weitere Dimensionen, die sich heute als Standard für die Untersuchung von Lebenslagen durchgesetzt haben (vgl. z.B. BMA 2001; Mühling 2005). Für diesen Beitrag werden vier soziale Indikatoren genutzt ${ }^{7}$ : das persönliche Einkommen, das Haushaltseinkommen, die Schulbildung und die Berufsausbildung. Damit sind neben der Einkommensarmut auch Teilhabechancen erfasst. In unserer Analyse steht die soziale Lage der Eltern im Vordergrund. Die ausgewählten Indikatoren lassen allerdings Rückschlüsse auf die Versorgungslage und das Wohlbefinden der Kinder zu.

Der DJI-Survey AID:A ist ferner vom Konzept der (familialen) Lebensführung geleitet (vgl. zum Beispiel Jürgens 2001; Jurczyk et al. 2009). Als Konzept, mit dem die alltägliche Koordinierung von Tätigkeiten des einzelnen und der Familienmitglieder gefasst wird, hat es sich bisher vor allem zur theoretischen Orientierung qualitativer Studien bewährt. Mit dem DJI-Survey AID:A liegt nun ein großer quantitativer Datensatz vor, der sich dem Alltag von Familien nähert. In diesem Beitrag werden aus diesem Variablenkomplex speziell die Zeit von Eltern für ihre Kinder, die Zufriedenheit der Eltern mit ihrer Zeitverwendung, die Sicherung eines günstigen Familienklimas und von Glück in der Paarbeziehung analysiert. Diese Auswahl wurde getroffen, um Hypothesen zu überprüfen, die sich aus dem vorliegenden Forschungsstand ergeben (vgl. 3.2 und 3.3). Da die Familiengründung Paaren oft Anlass zu einer Retraditionalisierung der innerfamilialen Arbeitsteilung bietet (Schulz/Blossfeld 2006), scheint eine geschlechtsspezifische Betrachtungsweise der Lebenslage, der Zeitverwendung und Zufriedenheit von Eltern unerlässlich.

\subsection{Zum Forschungsstand}

Es gibt in Deutschland eine ganze Reihe von Untersuchungen, die sich mit frühen und wenige, die sich mit späten Eltern befasst haben. Zusammen erzeugen sie ein disparates Wissen, das noch keine Vergleichbarkeit der Lebenslage, Lebensform und Lebensführung von Eltern gewährleistet, die zu unterschiedlichen Zeitpunkten eine Familie gründen. Dazu trägt auch bei, dass die Altersgrenzen für frühe und späte Elternschaft immer wieder anders gesetzt wurden und die Untersuchungen oft nur spezielle Teilpopulationen einbezogen, etwa solche, die über spezielle Bildungs- oder Hilfeeinrichtungen erreichbar waren. Zudem fällt auf, dass sich die meisten Untersuchungen auf die Mütter konzentrieren. Über die vom Timing der Familiengründung abhängige Lebenssituation und die Lebensführung früher, „mittlerer“ und später Väter wissen wir noch sehr wenig.

7 Auf die Nutzung weiterer Indikatoren für die soziale Lage der Eltern wurde hier verzichtet, um die Komplexität der Analyse zu beschränken. 
Was die sehr frühe Mutterschaft betrifft, ist bekannt, dass sie in Deutschland, etwa im Vergleich zu den USA, sehr selten ist: Die Zahl der Schwangerschaften speziell von 15bis 19-jährigen Frauen liegt in Deutschland mit 16 pro 1.000 Frauen im internationalen Vergleich (USA 84 pro 1.000) extrem niedrig (Schmidt 2009: 23; Spies 2010). Mehrere deutsche Studien verweisen auf eine zumeist sehr prekäre Lebenssituation minderjähriger Mütter (Friedrich/Remberg 2005; Spies 2009; Anslinger 2009; Matthiesen/Schmidt 2009; Block/Schmidt 2009). Das Sozialprofil minderjähriger Schwangerer und Mütter sowie ihrer Partner ist durch häufiger einfache Schulbildung, oft fehlende Berufsausbildungen und überdurchschnittlich häufige Arbeitslosigkeit geprägt (Matthiesen/Schmidt 2009; Block/ Schmidt 2009). An der zumeist prekären Situation minderjähriger Mütter sind kaum Zweifel angebracht. Ihre Entwicklungs- und Teilhabechancen sind zumindest in der Phase, in der ihre Kinder noch klein sind, gering. ${ }^{8}$ Die ungünstige ökonomische Situation junger Mütter ist allerdings nicht nur eine Folge früher Mütterschaft, sondern auch als deren Entstehungskontext von Relevanz (Bonell 2004; Friese 2008; Hobcraft 2008).

Über die frühen Väter weiß man in Deutschland sehr wenig. Einen Hinweis auf ihre Lebenssituation gibt die Untersuchung von Klindworth, Walter und Helfferich (2004). Hier zeigt sich, dass Männer, die früh ihr erstes Kind zeugten, überdurchschnittlich häufig nur eine kurze Schulbildung haben, sich früh in den Arbeitsmarkt integrieren und häufiger in Ostdeutschland leben. Weiterhin beeinflusst eine früh eingegangene Partnerschaft, ein konkreter Kinderwunsch und das nichteheliche Zusammenleben oder die Eheschließung vor dem 25. Lebensjahr die Wahrscheinlichkeit, „früh“ ein Kind zu zeugen (Klindworth et al. 2004). ${ }^{9}$

Während minderjährige Mütter relativ viel Interesse auf sich gezogen haben, wissen wir über die spezifische Lebenssituation der Mütter, die nach dem 18. Lebensjahr und dennoch vor dem Durchschnitt der Mütter ihr erstes Kind bekommen haben, noch wenig. Ihre Zahl geht von Jahr zu Jahr zurück (vgl. Statistisches Bundesamt 2010), ohne dass wir sagen können, unter welchen Bedingungen sie und ihre Partner mit ihren Kindern leben.

Relativ gut sind wir über die Gruppe der Eltern im Studium informiert. Sie sind allerdings keine altershomogene Gruppe (Cornelißen/Fox 2007; Middendorf 2008; Helfferich et al. 2007). Auch die Gruppe der späten Mütter hat Interesse auf sich gezogen (Herlyn/ Krüger 2003). Mütter, die ihr erstes Kind mit 35 Jahren oder später bekommen, so stellt Heinzelmann auf der Basis von Daten des Sozio-ökonomischen Panels 1998 fest, hätten im Durchschnitt deutlich häufiger Abitur oder Hochschulreife, seien beruflich besser qualifiziert als frühere Mütter und meist bis zur Mutterschaft berufstätig. Nach der Geburt verzichteten die späten Mütter häufiger auf eine Erwerbstätigkeit als die früheren. Überdurchschnittlich viele der späten Mütter seien verheiratet (Heinzelmann 2003: 65ff). Fosen-Schlichtinger stellt fest, dass späte Mütter, die ihr erstes Kind erst jenseits der 40

8 Aus pädagogischer Sicht wird die mangelnde gesellschaftliche Akzeptanz minderjähriger Mütter und die damit verbundene Ausgrenzung als kennzeichnend für die Situation dieser Mütter beschrieben (Spies 2010) und dafür plädiert, frühe Mutterschaft auch als Entwicklungschance für junge Frauen zu verstehen (Stauber 2010) und ihnen Ausbildungsangebote zu machen, deren Nutzung sie mit der Verantwortung für ein Kind vereinbaren können (Friese 2008).

9 Stauber macht zu Recht darauf aufmerksam, dass es auch innerhalb der frühen Mütter und Väter eine Varianz der Lebenslagen und Lebensformen gibt. Soziale Herkunft, Bildungsstand, sozioökonomische Lage und die Stabilität der Partnerschaft sind auch in dieser Gruppe nicht homogen (Stauber 2010: 88). 
bekämen, zufriedener seien, häufiger nach guter Ausbildung in ökonomischer Sicherheit und in einer stabilen Partnerschaft lebten. Sie könnten insgesamt ein Familienklima schaffen, das für ihre Kinder ,gesünder“ sei (Fosen-Schlichtinger 2006: 65). Auf eine amerikanische Befragung von spät geborenen Kindern rekurrierend (Yarrow 1991) hebt Fthenakis bezogen auf späte Väter hervor, dass auch sie ihren Kindern ökonomisch und affektivemotional relativ günstige Bedingungen böten, in stabileren Beziehung lebten, über größere berufliche und finanzielle Sicherheit verfügten und sich daraus eine größere Flexibilität in ihrer Zeitplanung ergäbe. Entsprechend könnten die späten Väter mehr Zeit mit ihren Kindern verbringen als andere (Yarrow 1991).

Es gibt also einige Anhaltspunkte für die Annahme, dass die Lebenslage, Lebensform und Lebensführung von Familien mit dem Timing der Familiengründung variiert. Allerdings sind die vorliegenden Befunde noch lückenhaft und auf Grund des kulturellen Zusammenhangs und des Zeitpunkts ihrer Erhebung auch nicht ohne weiteres auf das Deutschland von heute übertragbar. Die vorliegenden Befunde sind Ausgangspunkt für die folgenden Hypothesen.

\subsection{Hypothesen}

1. Frühe Eltern haben häufig nur eine einfache Schulbildung. Bei späterer Familiengründung verfügen Eltern über qualifiziertere Schul- und Berufsabschlüsse.

2. Frühe (Erst-)Elternschaft birgt hohe ökonomische Risiken. Eine spätere Familiengründung sichert Eltern und Kind eine zunehmend bessere ökonomische Situation.

3. Bei früher Familiengründung ist die Beziehung der Eltern weniger stabil. Bei späterer Familiengründung ist die Beziehung der Eltern stabiler und häufiger durch eine Ehe abgesichert. Die Kinder später Eltern leben häufiger mit beiden Elternteilen zusammen.

4. Die frühen Mütter und Väter sind häufiger als die „mittleren“ und späten noch nicht in den Arbeitsmarkt integriert. Die späten Mütter unterbrechen häufiger ihre Erwerbsarbeit nach der Geburt des ersten Kindes länger und geben sie häufiger ganz auf.

5. Sind die Eltern bei der Geburt ihres ersten Kindes bereits älter, so können sie ein günstigeres Familienklima schaffen. Späte Väter wie Mütter nehmen sich mehr Zeit für ihre Kinder.

\section{Zur Datengrundlage}

Wir greifen für unsere Analyse auf zwei unterschiedliche Datensätze zurück. Im Vordergrund stehen Daten der aktuellen Studie „Aufwachsen in Deutschland: Alltagswelten“ (AID:A), die 2009 am Deutschen Jugendinstitut durchgeführt wurde ${ }^{10}$. Zusätzlich werden Daten des Mikrozensus 2007 verwandt.

10 Im Jahre 2009 wurde deutschlandweit eine repräsentative Einwohnermeldeamtsstichprobe von etwa 25.000 Zielpersonen zwischen 0 und 55 Jahren gezogen. Die 9- bis 55-Jährigen wurde mit Hilfe von 
Der Mikrozensus 2007, der zum Zeitpunkt der Analyse jüngste allgemein zugängliche Datensatz des Mikrozensus hat den Vorteil, mit einer sehr großen Stichprobe, repräsentative Aussagen zu erlauben. Er eignet sich, die Verteilung spezifischer Gruppen in der Gesamtbevölkerung, auch relativ kleiner Gruppen, zutreffend zu schätzen. Bei der vorgegebenen Fragestellung erweisen sich allerdings zwei seiner Eigenschaften als größere Mängel: Erstens wird Elternschaft generell nach dem Koresidenzprinzip erfasst. D.h. Frauen und Männer sind nur dann als Eltern identifizierbar, wenn sie mit Kindern zusammen wohnen. So werden multilokale Familien gar nicht erfasst und manche Haushaltsgemeinschaft wird als Familie deklariert, ohne dass sie dies auch ihrer Blutsverwandtschaft oder ihrem Selbstverständnis gemäß ist. ${ }^{11}$ Zweitens findet man in den Mikrozensus-Daten nur sehr wenige Informationen zu relevanten Aspekten familialer Lebensführung und Zufriedenheit.

In den Daten von AID:A sind dagegen die leiblichen Kinder aller Befragten unabhängig davon, wo sie leben, erfasst. Für die Teilstichprobe der 18- bis 55-jährigen Befragten wurden die Lebenslage und vielfältige Aspekte familialer Lebensführung und Zufriedenheit erhoben. $\mathrm{Zu}$ berücksichtigen ist aber, dass AID:A als empirische Querschnittbefragung die typischen Eigenschaften und Selektivitäten von Surveys aufweist. Die befragten Mütter und Väter verfügen daher, im Vergleich zur Gesamtbevölkerung, über ein eher überdurchschnittliches Bildungsniveau und damit auch überdurchschnittliche finanzielle Ressourcen. Vor diesem Hintergrund müssen alle weiteren Ergebnisse betrachtet werden ${ }^{12}$.

\section{Befunde zur Lebenslage, Lebensform, Zeitverwendung und zum Wohlbefinden von Familien in Abhängigkeit vom biographischen Zeitpunkt der Familiengründung}

Im Folgenden werden Daten zur Lebenslage, Lebensform, Zeitverwendung und zum Wohlbefinden früher, „mittlerer“ und später Väter und Mütter aus dem DJI-Survey AID:A präsentiert und, soweit dies möglich ist mit dem Mikrozensus 2007 abgeglichen. Die Daten aus dem Mikrozensus finden sich überwiegend in den Fußnoten wieder.

computerassistierten Telefoninterviews (CATI) befragt. Für die jüngeren Zielpersonen antwortete ein Elternteil, meist die Mutter, ebenfalls im Telefoninterview.

11 Da Kinder nach der Trennung ihrer Eltern deutlich häufiger bei ihren Müttern als bei ihren Vätern leben, werden die Väter schlechter erfasst, evtl. werden Männern (auch manchen Frauen) Kinder zugeordnet, die nicht ihre leiblichen sind und für die sie auch sozial keinen Vater (bzw. keine Mutter) darstellen.

12 Insofern ist es wichtig zu prüfen, ob sich im Mikrozensus bei vergleichbaren Variablen die gleichen Unterschiede zwischen den Timing-Gruppen ergeben. 


\subsection{Lebenslage von Familien in Abhängigkeit vom biographischen Zeitpunkt der Familiengründung}

Wie dem vorliegenden Forschungsstand entsprechend zu erwarten war, unterscheiden sich die frühen Mütter in ausgewählten soziodemographischen Variablen wie Schulbildung, Einkommen und Lebensform deutlich von den späten Müttern, zum Teil aber auch signifikant von den „,mittleren“ Müttern ${ }^{13}$ (vgl. Tabelle A1 im Anhang) ${ }^{14}$. Ähnliches gilt für die Väter (vgl. Tabelle A2 im Anhang): Die drei Timing-Gruppen befinden sich in unterschiedlichen sozialen Lagen.

\section{Zur Schulbildung}

Die frühen Mütter haben häufiger als die späten Mütter nur einen Haupt- oder Realschulabschluss absolviert (vgl. Tabelle A1) ${ }^{15}$. Die mittleren Mütter liegen mit ihren Bildungsressourcen zwischen den beiden Extremgruppen ${ }^{16}$. Folgt man dem Forschungsstand, so kommen für diese Rangfolge drei Ursachen in Frage: Erstens: Jugendlichen mit schlechten Schulabschlüssen werden im Durchschnitt früher Eltern, zum Beispiel, weil sie weniger konsequent verhüten oder weil ihnen ihre fehlenden oder schlechten Schulabschlüsse wenig Perspektiven auf Anerkennung im beruflichen Bereich liefern. Unter diesen Umständen ist die Familiengründung eine alternative Strategie überhaupt einen Erwachsenenstatus zu erwerben (Friese 2008; Spies 2010). Zweitens: Mit ihrem jüngeren Alter ist bei frühen Eltern auf Grund der institutionalisierten Schulzeit eine geringere Chance verbunden, schon anspruchsvolle Abschlüsse gemacht zu haben. Drittens: Eine begonnene Ausbildung kann nach der Familiengründung nicht ohne weiteres fortgesetzt werden (Anslinger 2009; Cornelißen/Fox 2007; Friese 2008).

Die späten Mütter haben in der Familiengründungsphase erwartungsgemäß die anspruchsvollsten Bildungsabschlüsse, welche langfristig mit höheren Erwerbs- und Einkommenschancen einhergehen. Bei den Vätern zeichnet sich die gleiche Tendenz ab. ${ }^{17}$

13 Gerechnet wurden mit den AID:A-Daten für jedes Geschlecht zwei multinomiale logistische Regressionen, bei denen einmal jeweils die frühen Mütter bzw. Väter die Referenzkategorie darstellten und einmal die späten Mütter bzw. Väter.

14 Zur Interpretation der multinomial logistischen Regression müssen bei kategorialen unabhängigen Variablen zwei Referenzkategorien berücksichtigt werden, also eine Kategorie der abhängigen Variable und eine Kategorie der unabhängigen Variable (im Modell durch 0 gekennzeichnet). Die exakte Interpretation erfolgt folgendermaßen: Die Chance, eher den frühen als den späten Müttern anzugehören, ist bei einer Person mit Mittlerer Reife, gegenüber einer Person mit Abitur, 3,92-fach größer.

15 In der multinomial logistischen Regression zeigen sich, gegen die Referenzkategorie „Abitur“ getestet, signifikante Effekte nur bei der ,„,Mittleren Reife“.

16 Dies geht aus einem zweiten Modell mit der Referenzkategorie „frühe Mütter“ hervor. Es ermöglicht einen direkten Vergleich der ,mittleren“ mit den frühen Müttern (ohne Abbildung).

17 Auch mit den Mikrozensusdaten lässt sich die erste Hypothese bestätigen: Laut Mikrozensus haben frühe Mütter häufiger, nämlich in 40,5\% aller Fälle nur einen Hauptschulabschluss. Unter den Müttern, die ihr erstes Kind später bekommen, ist dieser Anteil deutlich geringer, bei den späten Müttern, die nach dem 32. Lebensjahr ihr erstes Kind bekamen, liegt er bei 18,6\%. Umgekehrt ist der Anteil der Mütter mit Hochschulreife bei den frühen Müttern mit 9,5\% sehr gering. Er steigt mit dem Alter der Mütter bei der Geburt ihres ersten Kindes und liegt bei den späten Müttern bei 39,2\%. 
Auch die frühen Väter haben, verglichen mit den späten, ein niedrigeres Bildungsniveau zu verzeichnen und häufiger als diese eine Haupt- oder Realschule abgeschlossen (vgl. Tabelle A2). Die mittleren Väter hingegen unterscheiden sich nicht signifikant von den späten Vätern. Beide Gruppen sind, ihre Bildungsressourcen betreffend, ähnlich ausgestattet und setzen sich hierdurch von den frühen Vätern $a b .{ }^{18}$ Insgesamt haben sich die Väter zum Zeitpunkt der Familiengründung bessere Startvoraussetzungen für das Erwerbsleben geschaffen als die Mütter. Dies dürfte eine Folge der im Durchschnitt biografisch früheren Familiengründung von Frauen sein, macht aber auch deutlich, wie sehr gerade bei Männern die Norm verankert ist, eine Familiengründung erst nach Abschluss von Schule und Ausbildung zu verwirklichen (vgl. Zerle/Krok 2008). Die ungleichen Startchancen dürften nicht selten für die Arbeitsteilung der Eltern relevant werden.

\section{Zum Einkommen und zur Erwerbsbeteiligung}

Hatten schon die Untersuchungen zu den Schwangerschaften von Müttern unter 18 Jahren gezeigt, dass die Situation von sehr frühen Müttern aus ökonomischer Sicht ausgesprochen prekär ist, so zeigt sich auch in den AID:A-Daten ein signifikanter Zusammenhang zwischen dem Alter der Mütter bei der Geburt ihres ersten Kindes und dem persönlichen sowie dem Haushaltsnettoeinkommen (vgl. Tabelle A1): Die frühen Mütter haben häufiger als die späten Mütter kein eigenes Einkommen oder ein geringeres persönliches Nettoeinkommen. Auch das Nettohaushaltseinkommen der frühen Mütter liegt niedriger. ${ }^{19}$ Die Einkommen der „mittleren“ Mütter liegen ebenfalls unter denen der späten Mütter, die „,mittleren“ Mütter stehen jedoch finanziell besser da als die frühen Mütter. Auch die Finanzausstattung der frühen Väter liegt unter jener der mittleren und der späten Väter: Die frühen Väter haben ein geringeres persönliches Nettoeinkommen als die späten Väter; die „mittleren“ Väter und die späten Väter verfügen über ähnliche Einkommen. Was das Nettohaushaltseinkommen betrifft, liegt das der frühen und „mittleren“ Väter unter dem der späten Väter. Die deskriptiven Befunde legen nahe, dass das geringe Haushaltseinkommen der frühen Eltern wesentlich durch den hohen Anteil $(22,2 \%)$ an frühen Müttern bedingt ist, die über kein eigenes Einkommen verfügen (vgl. Tabelle 2).

Ähnlich variiert in den Mikrozensusdaten auch die Schulbildung der Väter in der Familiengründungsphase mit dem Zeitpunkt ihrer ersten Vaterschaft.

18 Das zweite Modell, das den Vergleich der „mittleren“ mit den frühen Vätern ermöglicht (ohne Abbildung) zeigt, dass sich die ,mittleren“ Väter signifikant von den frühen Vätern unterscheiden. Im Mikrozensus wird deutlich, dass auch unter den Vätern die erwartete Rangordnung der TimingGruppen zu finden ist und sich die „mittleren“ von den späten Vätern weniger unterscheiden, als die frühen und die „mittleren“. Unter den frühen Vätern haben 41,2\% nur einen Hauptschulabschluss und nur 14,3\% eine Hochschulreife unter den „mittleren“ Vätern haben 36,7\% nur einen Hauptschulabschluss und 31,6\% eine Hochschulreife, unter den späten haben sich nur 26,7\% auf einen Hauptschulabschluss beschränkt und 36,2\% haben das (Fach-)Abitur.

19 Im Mikrozensus 2007 zeigt sich ebenfalls ein signifikanter Zusammenhang zwischen dem Alter der Eltern bei der Geburt ihres ersten Kindes und dem Haushaltseinkommen. Wenn Mütter früh mit der Familiengründung beginnen, so hat dies für den Lebensstandard ihres Kindes noch deutlich häufiger gravierende Folgen als wenn Väter verhältnismäßig früh eine Familie gründen. 22,5\% der frühen Mütter lebt mit einem Haushaltseinkommen unter $1.100 €$. Dasselbe gilt für 7,4\% der ,,mittleren“ Mütter und für 5,5\% der späten. Bei den Vätern besteht dieser Zusammenhang in den Mikrozensusdaten auch; allerdings liegt selbst bei den frühen Vätern der Anteil derer, die mit einem Haushaltseinkommen unter $1.100 €$ leben, nur bei $7,7 \%$, bei den späten bei nur $2,2 \%$. 
Tabelle 2: Persönliches Nettoeinkommen früher, mittlerer und später Eltern

\begin{tabular}{|c|c|c|c|c|c|c|}
\hline & $\begin{array}{l}\text { Frühe } \\
18-24\end{array}$ & $\begin{array}{c}\text { Mütter } \\
\text { Mittlere } \\
\text { 25-32 }\end{array}$ & $\begin{array}{c}\text { Späte } \\
33+\end{array}$ & $\begin{array}{c}\text { Frühe } \\
18-29\end{array}$ & $\begin{array}{c}\text { Väter } \\
\text { Mittlere } \\
\text { 29-35 }\end{array}$ & $\begin{array}{c}\text { Späte } \\
36+\end{array}$ \\
\hline kein eigenes Einkommen & 22,2 & 18,1 & 7,1 & 2,3 & 0,5 & 1,1 \\
\hline unter 1000 Euro & 58,1 & 51,7 & 43,8 & 19,9 & 4,9 & 2,1 \\
\hline 1000-1999 Euro & 17,1 & 24,5 & 32,1 & 48,3 & 32,8 & 26,3 \\
\hline 2000 Euro und mehr & 2,6 & 5,7 & 17,0 & 29,5 & 61,7 & 70,5 \\
\hline Gesamt & 100 & 100 & 100 & 100 & 100 & 100 \\
\hline$(\mathrm{N})$ & $(117)$ & $(441)$ & $(112)$ & $(176)$ & $(183)$ & (95) \\
\hline
\end{tabular}

Quelle: AID:A - DJI-Survey 2009; 18- bis 55-Jährige mit leiblichen Kindern unter 7 Jahren; N=1.124 (ungewichtete Daten); Angaben in Prozent

Auch mittlere Mütter haben häufiger kein eigenes Einkommen. Bei den späten Müttern hingegen tragen nur 7,1\% gar nicht zum Familieneinkommen bei. Insgesamt verfügen die frühen und „mittleren“ Mütter über geringere Einkommen als die späten. Auch bei den Vätern zeigen sich zwischen den Timing-Gruppen große Unterschiede: Zwar finden sich in allen Gruppen kaum Väter ohne eigenes Einkommen, aber die Anteile der Väter mit höheren Einkommen steigen über die Gruppen hinweg stark an. Der Geschlechtervergleich zeigt, dass in jeder Altersgruppe deutlich mehr Mütter als Väter gänzlich ohne eigenes Einkommen leben. Diese Diskrepanzen weisen auf die relativ traditionelle Arbeitsteilung in Haushalten hin, in denen nur Kinder unter sieben Jahren leben. Zum Befragungszeitpunkt sind $60 \%$ der frühen Mütter und $41,2 \%$ der späten nicht erwerbstätig. Damit bestätigt sich die Hypothese, die nach Befunden von Heinzelmann mit Daten von 1998 aufgestellt wurde, nicht: Späte Mütter sind heute in der Familiengründungsphase besser in den Arbeitsmarkt integriert als frühe und mittlere Mütter. ${ }^{20}$

Die Erwerbsquoten der Väter liegen, gleich in welcher Timing-Gruppe, deutlich über denen der Mütter. Gleichzeitig erweist sich die Situation der frühen Väter als besonders ungünstig: Mit 25,4\% ist - gemessen an den anderen Vätern - ein verhältnismäßig großer Anteil der frühen Väter zum Befragungszeitpunkt nicht erwerbstätig. Die Situation der frühen Mütter ist also zweifellos besonders prekär, aber auch die frühen Väter sind noch vergleichsweise wenig ökonomisch abgesichert und im Vergleich zu den anderen Vätern noch verhältnismäßig schlecht in den Arbeitsmarkt integriert. Da ein hoher Prozentsatz der ersten Kinder früher Mütter auch einen frühen Vater hat, nämlich 69\%, kumulieren bei einem großen Teil der jungen Familien ökonomische Risikolagen und ungünstige Erwerbschancen.

\section{Zum Familienstand und zur Form des Zusammenlebens}

Wie zu erwarten war, sind in biografisch früh gegründeten Familien die Beziehungen der Eltern weniger stabil und seltener durch eine Ehe institutionalisiert. Laut Mikrozensus 2007 sind 38\% der frühen Mütter und 24\% der frühen Väter bis zur Einschulung ihres ersten Kindes noch ledig. Dasselbe gilt nur für 17\% der späten Mütter und 10\% der späten

20 Die Daten des Mikrozensus zeigen ebenfalls, dass die späten Mütter in der Familiengründungsphase häufiger als die jüngeren einer Beschäftigung nachgehen. Ihre Erwerbsquote liegt hier bei $52 \%$, die der ,mittleren“ Mütter bei 49\%, die der frühen bei nur 33\%. 
Väter. In den AID:A-Daten zeigt sich der wenig stabile Charakter der Paarbeziehungen früher Eltern auch darin, dass ein Viertel der frühen Mütter und Väter zum Befragungszeitpunkt getrennt vom anderen Elternteil ihres ersten Kindes lebt (vgl. Tabelle 3). Zwischen den Geschlechtern zeigen sich in dieser Hinsicht also keine Unterschiede. Es zeigt sich aber, dass die Mütter und Väter, die ihr erstes Kind später bekommen haben, deutlich häufiger noch mit dem anderen Elternteil ihres ersten Kindes zusammen leben. Die Daten bestätigen also die dritte Hypothese.

Tabelle 3: Lebensformen früher, mittlerer und später Eltern

\begin{tabular}{|c|c|c|c|c|c|c|}
\hline Prozentualer Anteil derer, die... & $\begin{array}{l}\text { Frühe } \\
18-24\end{array}$ & $\begin{array}{c}\text { Mütter } \\
\text { Mittlere } \\
\text { 25-32 } \\
\end{array}$ & $\begin{array}{c}\text { Späte } \\
33+\end{array}$ & $\begin{array}{c}\text { Frühe } \\
18-29 \\
\end{array}$ & $\begin{array}{c}\text { Väter } \\
\text { Mittlere } \\
29-35 \\
\end{array}$ & $\begin{array}{c}\text { Späte } \\
36+\end{array}$ \\
\hline ... noch mit dem anderen Elternteil zusammen sind & 74,6 & 94,8 & 95,1 & 76,9 & 99,2 & 97,6 \\
\hline ... mit einem Partner im Haushalt leben & 75,0 & 94,5 & 94,0 & 76,9 & 98,1 & 94,9 \\
\hline ... mit dem Kind im gemeinsamen Haushalt leben & 100 & 100 & 99,2 & 74,4 & 97,3 & 94,3 \\
\hline $\mathbf{N}$ & 124 & 478 & 133 & 117 & 264 & 174 \\
\hline
\end{tabular}

Quelle: AID:A - DJI-Survey 2009; 18- bis 55-Jährige mit leiblichen Kindern unter 7 Jahren; N=1.290 (gewichtet auf 18-55); Angaben in Prozent

Für die Kinder früher Eltern bedeutet dies, dass sie seltener als die Kinder anderer TimingGruppen die Gelegenheit haben, mit beiden Elternteilen zusammen aufzuwachsen. Sie bleiben in der hier beschriebenen Familienphase fast immer bei den Müttern. Fast ein Viertel der frühen Väter lebt schon bald nach der Geburt ihres ersten Kindes nicht mehr mit diesem zusammen (vgl. Tabelle 3).

Wie erwartet, variieren mit dem Timing der Erstelternschaft wichtige Aspekte der sozialen Lage von Eltern und ihren Kindern. Dabei erweist sich die Situation früher Eltern als weniger gefestigt. Schon wenn das Timing dem Durchschnitt entspricht, nicht erst bei später Elternschaft, befinden sich die Familien zum großen Teil in einer vergleichsweise stabilen Situation,

\subsection{Zeitverwendung von Eltern in Abhängigkeit vom biographischen Zeitpunkt der Familiengründung}

Viele Studien - am deutlichsten jedoch die diversen Auswertungen der Zeitbudgeterhebungen des Statistischen Bundeamtes - haben belegt, dass sich die Zeitverwendung von Frauen und Männern für verschiedene Aufgabenbereiche seit den 90er Jahren des letzten Jahrhunderts leicht angeglichen hat. Frauen leisten heute etwas mehr Erwerbsarbeit als Anfang der 1990er Jahre und sie haben den Umfang ihrer Haus- und Sorgearbeit etwas reduziert. Die Zeitbudgets der Männer haben sich nur minimal gewandelt (Statistisches Bundesamt 2003: 14). In Paarhaushalten mit Kindern leisteten Männer eine Stunde und 9 Minuten Kinderbetreuung als Hauptaktivität, Frauen in Paarhaushalten dagegen 2 Stunden 43 Minuten und alleinerziehende Frauen gut drei Stunden (Statistisches Bundesamt 2003: 22). Bezogen auf die Kinderbetreuung als gleichzeitige Aktivität neben anderen scheint die Arbeitsteilung in Paarbeziehungen noch deutlicher geschlechtsspezifisch geprägt. In dieser Lebensform verbringen Frauen dreimal so viel Zeit wie Männer mit ihren Kindern (ibd.). 
Hier wird nun betrachtet, was frühe und späte Mütter und Väter im AID:A-Survey erstens über den (geschätzten) zeitlichen Umfang ihrer aktiven Zeit mit ihrem Kind bzw. den Kindern berichten. Zweitens wird auf die Zufriedenheit der Eltern mit der Zeitverwendung in relevanten Bereichen der Work-Life-Balance eingegangen und drittens wird das subjektive Wohlbefinden der Befragten in den Blick genommen. Bei dieser Analyse ist zu beachten, dass die Zeit für Kinder durch die Erwerbszeiten von Eltern strukturiert und limitiert wird. Dabei schwanken die Erwerbsquoten der Väter laut Mikrozensus zwischen $85 \%$ und 94\%, die der Mütter zwischen 33\% und 52\%. Die frühen Väter und Mütter weisen im Vergleich zu den anderen Timing-Gruppen des gleichen Geschlechtes jeweils die geringsten Erwerbsquoten auf, unter den Müttern sind die späten (!) und unter den Vätern die „mittleren“ am häufigsten erwerbstätig. Während die Teilzeitquote der erwerbstätigen Väter zwischen 5\% und 8\% liegt, liegt sie bei den Müttern unabhängig vom Timing der Mutterschaft deutlich höher, nämlich bei $57 \%$ bzw. $58 \%$.

\section{Zeitverwendung mit den Kindern}

Die Daten zur Zeitverwendung mit den Kindern bestätigen den Sonntag eindeutig als Familientag (vgl. Tab. 4): die Mütter und Väter verbringen dann sehr viel Zeit mit ihren Kindern - und das relativ unabhängig vom Timing der Familiengründung. 60 bis 70\% der Mütter und 50 bis $60 \%$ der Väter sind an Sonntagen „mehr als 5 Stunden“ mit ihren Kindern aktiv; zwischen 30 und 40\% der Eltern verbringen nach eigenen Angaben zumindest „3 bis 5 Stunden“ mit ihnen. Wesentliche Geschlechterunterschiede zeigen sich nicht; keinesfalls kompensieren die Väter aber ihre Zeitknappheit während der Woche mit einem ausgiebigeren Zeitvertreib mit den Kindern am Wochenende. Bei Vollzeit erwerbstätigen Müttern hingegen (v.a. den späten Müttern) scheint dies anders: Verglichen mit dem Durchschnitt der Mütter schätzen sie sich als aktiver ein und übertreffen das Engagement der Väter deutlich. Bis zu 90\% geben an, an Sonntagen „mehr als 5 Stunden“ mit ihren Kindern aktiv zu sein ${ }^{21}$.

Tabelle 4: Wie viele Stunden beschäftigen Sie sich an Sonntagen aktiv mit Ihrem Kind?

\begin{tabular}{lrrrrrr}
\hline & \multicolumn{3}{c}{ Mütter } & \multicolumn{3}{c}{ Väter } \\
& $\begin{array}{r}\text { Frühe } \\
\text { 18-24 }\end{array}$ & $\begin{array}{c}\text { Mittlere } \\
\mathbf{2 5 - 3 2}\end{array}$ & $\begin{array}{c}\text { Späte } \\
\mathbf{3 3 +}\end{array}$ & $\begin{array}{c}\text { Frühe } \\
\mathbf{1 8 - 2 9}\end{array}$ & $\begin{array}{c}\text { Mittlere } \\
\mathbf{2 9 - 3 5}\end{array}$ & $\begin{array}{c}\text { Späte } \\
\mathbf{3 6 +}\end{array}$ \\
\hline Aktiv an Sonntagen & & & & & & \\
Gar nicht & - & - & - & 1,1 & 0,4 & - \\
Circa eine halbe Stunde & - & - & - & - & - & - \\
Circa eine Stunde & - & - & - & 4,6 & 0,4 & - \\
1 bis 2 Stunden & 5,8 & 4,8 & 3,7 & 5,7 & 3,9 & 3,1 \\
3 bis 5 Stunden & 31,9 & 31,1 & 28,0 & 41,4 & 36,0 & 36,4 \\
Mehr als 5 Stunden & 62,3 & 64,2 & 68,3 & 47,1 & 59,3 & 60,5 \\
\hline Gesamt & 100 & 100 & 100 & 100 & 100 & 100 \\
N & 70 & 399 & 243 & 86 & 257 & 164 \\
\hline
\end{tabular}

Quelle: AID:A - DJI-Survey 2009; 18- bis 55-Jährige mit leiblichen Kindern unter 7 Jahren in einem gemeinsamen Haushalt mit dem Befragten; $\mathrm{N}=1.219$ (gewichtet auf 18-55); Angaben in Prozent

21 Ohne Abbildung. Diese Werte können nur als Hinweis interpretiert werden. Für eine tiefergehende Analyse reichen hier die Fallzahlen nicht aus. 
Werktags sind die Mütter aktiver als die Väter: Während in allen drei Timing-Gruppen ein Drittel der Mütter selbst an Werktagen „mehr als 5 Stunden“ aktiv mit dem Kind verbringt, trifft dies nur auf bestenfalls $10 \%$ der Väter zu (vgl. Tabelle 5). Etwa einem Viertel der Mütter und der Hälfte der Väter bleiben unter der Woche nur weniger als zwei gemeinsam aktiv verbrachte Stunden mit ihrem Kind. Verglichen mit der täglichen Zeit am Arbeitsplatz ist das wenig. Dennoch ist der Umfang des berichteten Engagements während der Woche auch für die Väter als sehr hoch einzuschätzen - immerhin arbeiten etwa $90 \%$ der „mittleren“ und späten Väter und $70 \%$ der frühen Väter in Vollzeit. Nach der Arbeit finden knapp 30 bis $40 \%$ der Väter werktags noch die Zeit, sich „,mehr als 3 Stunden" aktiv mit dem Kind zu beschäftigen. Dies entspricht den Befunden von Grunow (2007), die eine starke Ausweitung des Engagements der Väter an den Wochenenden und nach Feierabend sieht.

Tabelle 5: Zeit mit und für Kinder in Abhängigkeit vom Timing der (Erst-)Elternschaft

\begin{tabular}{|c|c|c|c|c|c|c|}
\hline & $\begin{array}{l}\text { Frühe } \\
18-24\end{array}$ & $\begin{array}{c}\text { Mütter } \\
\text { Mittlere } \\
\text { 25-32 }\end{array}$ & $\begin{array}{c}\text { Späte } \\
33+\end{array}$ & $\begin{array}{c}\text { Frühe } \\
18-29\end{array}$ & $\begin{array}{c}\text { Väter } \\
\text { Mittlere } \\
\text { 29-35 }\end{array}$ & $\begin{array}{c}\text { Späte } \\
36+\end{array}$ \\
\hline \multicolumn{7}{|l|}{ Aktiv an Werktagen } \\
\hline Gar nicht & - & - & - & 1,2 & 0,4 & 0,0 \\
\hline Circa eine halbe Stunde & - & 1,0 & 0,8 & 3,5 & 8,6 & 4,9 \\
\hline Circa eine Stunde & 7,1 & 4,5 & 1,6 & 17,4 & 13,6 & 18,9 \\
\hline 1 bis 2 Stunden & 20,0 & 19,5 & 20,6 & 37,2 & 49,8 & 42,1 \\
\hline 3 bis 5 Stunden & 37,1 & 37,3 & 42,8 & 33,7 & 23,0 & 23,8 \\
\hline Mehr als 5 Stunden & 35,7 & 37,6 & 34,2 & 7,0 & 4,7 & 10,4 \\
\hline Gesamt & 100 & 100 & 100 & 100 & 100 & 100 \\
\hline $\mathrm{N}$ & 70 & 86 & 399 & 257 & 243 & 164 \\
\hline
\end{tabular}

Quelle: AID:A - DJI-Survey 2009; 18- bis 55-Jährige mit leiblichen Kindern unter 7 Jahren in einem gemeinsamen Haushalt mit dem Befragten; $\mathrm{N}=1.219$ (gewichtet auf 18-55); Angaben in Prozent

Vergleicht man ausschließlich das werktägliche Engagement Vollzeit erwerbstätiger Mütter mit dem der Väter für ihre Kinder, verringert sich der Unterschied zwischen den Geschlechtern maßgeblich: Die Mütter sind ähnlich selten wie die Väter in der Kategorie „mehr als fünf Stunden“ zu finden, verbringen aber dennoch häufiger als die Väter zumindest „drei bis fünf Stunden“ am Tag mit den Kindern. Trotz voller Erwerbseinbindung scheinen die Mütter auch an Werktagen engagierter zu sein. ${ }^{22}$ Dies muss kein unmittelbarer Effekt eines geschlechtsspezifischen Verständnisses von Elternschaft sein, sondern kann zumindest auch ein Effekt der längeren Arbeitszeit Vollzeit erwerbstätiger Väter im Vergleich zu Vollzeit erwerbstätigen Müttern sein, denn Vollzeit erwerbstätige Väter arbeiten, den AID:A-Daten zufolge, im Mittel vier Stunden pro Woche mehr als Vollzeit erwerbstätige Mütter.

Das zeitliche Engagement der Eltern für ihre Kindern erweist sich als weitgehend unabhängig vom Timing der ersten Elternschaft, zeigt sich aber als Resultat des Umfangs der Erwerbsarbeit. Die geringere berufliche Einbindung früher Mütter und Väter führt nicht zu einem höheren Zeitbudget von frühen Eltern für ihre Kinder. Die Hypothese,

22 Ohne Abbildung. Auch dies ist aufgrund der geringen Fallzahlen nur als Hinweis zu werten. 
dass späte Eltern mehr Zeit mit ihren Kindern verbringen als frühe, trifft allenfalls für die Zeitverwendung an Wochenenden zu, lässt sich für die Werktage aber nicht bestätigen.

\section{Zufriedenheit mit der Zeitverwendung}

Frühe, „mittlere“ und späte Eltern sind ähnlich zufrieden mit der Zeitverwendung für ihre Kinder, aber es gibt deutliche geschlechtsspezifische Unterschiede: Nur 13 bis 20\% der Mütter geben an, zu wenig Zeit mit ihren Kindern zu verbringen (vgl. Tab. 6). Bei den Vätern ist das Niveau der Unzufriedenheit deutlich höher und liegt zwischen 40 und 50\%. Es entspricht etwa der Zeitknappheit, welche von Vollzeit erwerbstätigen Müttern berichtet wird. So erhöhen sich die Anteile der (mit ihrer Zeit für Kinder) unzufriedenen Mütter auf 30 bis $45 \%$, wenn die Unterschiede im Erwerbsumfang zwischen den Geschlechtern konstant gehalten werden.

$\mathrm{Zu}$ viel Zeit wenden die Eltern ihrer Ansicht nach für den Beruf auf: Während der Anteil an allen Müttern, die angeben, zu viel Zeit für den Beruf zu verwenden, zwischen 15 und 34\% liegt, sind es bei den Vollzeit erwerbstätigen Müttern zwischen 30 und 60\%mit einem deutlichen Unterschied zwischen den frühen und „mittleren“ Müttern einerseits und den späten Müttern andererseits. Möglicherweise haben die späten Mütter wegen ihrer tendenziell höheren Bildung Tätigkeiten, die mit mehr Überstunden einhergehen. Auch bei den Vätern gibt ein hoher Anteil (30 bis $42 \%$ ) an, zu viel Zeit für den Beruf zu verwenden ${ }^{23}$. Über zu wenig Zeit für den Partner berichten 43 bis $70 \%$ der Eltern. Dabei sind die späten Eltern tendenziell unzufriedener und die Mütter unzufriedener als die Vä$\operatorname{ter}^{24}$. Zu knapp scheint den Eltern die persönliche Freizeit und die Zeit für die Freunde zu werden: Ein enorm hoher Anteil von 60 bis $76 \%$ gibt an, zu wenig Zeit für diese Bereiche zu verwenden. Auch hierbei sind die späten Eltern etwas unzufriedener als die frühen Eltern. Mit dem persönlichen Freizeitbudget sind die späten Väter weniger zufrieden als die späten Mütter, jedoch zufriedener als die Vollzeit erwerbstätigen Mütter.

Tabelle 6: Zufriedenheit mit der Zeitverwendung in ausgewählten Bereichen der WorkLife-Balance

\begin{tabular}{|c|c|c|c|c|c|c|}
\hline Prozentualer Anteil an... & $\begin{array}{c}\text { Frühe } \\
18-24\end{array}$ & $\begin{array}{c}\text { Mütter } \\
\text { Mittlere } \\
\text { 25-32 }\end{array}$ & $\begin{array}{c}\text { Späte } \\
33+\end{array}$ & $\begin{array}{l}\text { Frühe } \\
18-29\end{array}$ & $\begin{array}{c}\text { Väter } \\
\text { Mittlere } \\
\text { 29-35 }\end{array}$ & $\begin{array}{c}\text { Späte } \\
36+\end{array}$ \\
\hline ... zu wenig Zeit für die Kinder & 20,0 & 13,0 & 17,5 & 46,0 & 48,7 & 41,7 \\
\hline$\ldots$ zu viel Zeit für den Beruf & 15,6 & 12,8 & 33,6 & 29,5 & 42,1 & 42,1 \\
\hline ... zu wenig Zeit für den Partner & 45,6 & 56,7 & 69,4 & 43,0 & 46,2 & 49,4 \\
\hline ... zu wenig persönliche Freizeit & 64,3 & 72,4 & 74,0 & 58,0 & 72,1 & 76,4 \\
\hline$\ldots$ zu wenig Zeit für die Freunde & 64,3 & 62,3 & 65,7 & 60,9 & 63,0 & 75,9 \\
\hline $\mathrm{N}$ je Zelle für $100 \%$ & 70 & 87 & 399 & 263 & 246 & 175 \\
\hline
\end{tabular}

Quelle: AID:A - DJI-Survey 2009; 18- bis 55-Jährige mit leiblichen Kindern unter 7 Jahren in einem gemeinsamen Haushalt mit dem Befragten; N=1.240 (gewichtet auf 18-55); Angaben in Prozent

23 Die Unterschiede zwischen den Timing-Gruppen sind auf dem 5\%-Niveau nicht signifikant.

24 Betrachtet man nur die Vollzeit erwerbstätigen Mütter erhöht sich der Zusammenhang noch etwas, ist aber wegen der geringen Fallzahlen nicht mehr signifikant. 
Insgesamt lässt sich resümieren, dass in allen Timing-Gruppen die Zeit, die für den Beruf aufgewendet wird, vor allem bei den Vollzeit erwerbstätigen Müttern und Vätern, häufig als zu viel, die Zeit für die Kinder hingegen eher als zu wenig eingeschätzt wird. Am unzufriedensten sind Eltern jedoch mit ihrer zu knappen persönlichen Freizeit und ihrer Zeit für Freunde. Späte Mütter vermissen sehr häufig auch Zeit für ihren Partner.

\section{Wohlbefinden}

Familie und Paarbeziehungen leisten einen wichtigen Beitrag zur emotionalen Stabilisierung von Menschen, können aber auch ein Ort emotionaler Belastung sein (vgl. Jurczyk et al. 2009). Dem Wohlbefinden in Familie und Partnerschaft gilt daher in der Analyse familialer Lebensführung besondere Aufmerksamkeit. Das durchschnittliche Familienklima ${ }^{25}$ liegt in den für AID:A befragten Familien mit einem Mittelwert ${ }^{26}$ von 1,7 bei den Müttern und 1,6 bei den Vätern sehr hoch. Es wird von den frühen Eltern genauso gut bewertet wie von den späten Eltern. Den frühen Eltern, die zusammen bleiben, gelingt es offenbar genauso gut wie den späten, ein für sie angenehmes Familienklima zu erzeugen (vgl. Tab. 7).

Tabelle 7: Wohlbefinden von frühen, „mittleren“ und späten Eltern (Mittelwerte aus einer einfaktoriellen Varianzanalyse)

\begin{tabular}{lcccccc}
\hline & $\begin{array}{c}\text { Mütter } \\
\text { Frühe } \\
\mathbf{1 8 - 2 4}\end{array}$ & $\begin{array}{c}\text { Mittlere } \\
\mathbf{2 5 - 3 2}\end{array}$ & $\begin{array}{c}\text { Späte } \\
\mathbf{3 3 +}\end{array}$ & $\begin{array}{c}\text { Frühe } \\
\mathbf{1 8 - 2 9}\end{array}$ & $\begin{array}{c}\text { Väter } \\
\text { Mittlere } \\
\mathbf{2 9 - 3 5}\end{array}$ & $\begin{array}{c}\text { Späte } \\
\mathbf{3 6 +}\end{array}$ \\
\hline Mittelwerte & & & & & & \\
Familienklima & 1,7 & 1,6 & 1,7 & 1,6 & 1,6 & 1,7 \\
Glück in der Partnerschaft & 1,8 & 1,6 & 1,8 & 1,6 & 1,5 & 1,7 \\
\hline N je Zelle für 100\% & & & & & & \\
\hline
\end{tabular}

Quelle: AID:A - DJI-Survey 2009; 18- bis 55-Jährige mit leiblichen Kindern unter 7 Jahren; N=1.239 (gewichtet auf 18-55)

Dies zeigt sich auch in der Wahrnehmung der eigenen Partnerschaft. Auch diese wird von allen Timing-Gruppen insgesamt als sehr positiv wahrgenommen und die Mittelwerte ${ }^{27}$ liegen mit 1,7 bei den Müttern und 1,6 bei den Vätern sehr hoch. Gestritten wird nur bei 10 bis $15 \%$ der Paare häufig, wobei es den Paaren nach eigenen Angaben meist leicht fällt, eine Lösung für das Problem zu finden. Damit zeigen sich in Bezug auf die Partnerschaftsqualität, sowohl bei den Müttern als auch bei den Vätern, keine signifikanten Unterschiede in Abhängigkeit von dem Timing der ersten Elternschaft.

25 In AID:A wurde eine spezielle Skala zum Familienklima (Engfer 1978) abgefragt, die aus fünf einzelnen Items besteht: „Ich bin gerne mit meiner Familie zusammen“, „In unserer Familie können wir über alles sprechen“, „In unserer Familie geht jeder seinen eigenen Weg“, „In unserer Familie kommt es zu Reibereien" und „In unserer Familie haben wir viel Spaß miteinander".

26 Auf einer Skala, die Werte zwischen 1 (bedeutet ein sehr gutes Klima) und vier (bedeutet ein schlechtes Klima) annehmen kann.

27 Auf einer Skala, die Werte zwischen 1 (sehr glücklich) und 6 (sehr unglücklich) annehmen kann. 


\section{Fazit}

Mit dem Rückgriff auf zwei sehr unterschiedliche Datensätze war es möglich zu zeigen, wie die Lebenslage und Lebensführung von Familien mit dem Zeitpunkt der Familiengründung variiert. Mit Bezug auf die eingangs formulierten Hypothesen lässt sich festhalten: Frühe Elternschaft geht - auch wenn man diese Timing-Gruppe nicht so eng fasst wie bei den Untersuchungen über minderjährige Mütter - mit einer schlechten Ressourcenausstattung, wie einem einfachen Bildungsniveau, einer schlechteren Einbindung in den Arbeitsmarkt und damit auch einer schlechteren ökonomischen Situation einher. Die Konsumspielräume und Teilhabechancen früher Eltern (und ihrer Kinder) sind damit geringer als die anderer Timing-Gruppen. Auch die Paar- und Familienkonstellation früher Eltern ist weniger stabil. Frühe Eltern sind seltener verheiratet und leben häufiger vom anderen Elternteil ihres ältesten leiblichen Kindes getrennt. Die Kinder früher Eltern haben also seltener Gelegenheit, mit beiden Eltern gemeinsam aufzuwachsen.

Weitgehend unabhängig vom Timing der Familiengründung zeigen sich die aktiv mit den Kindern verbrachte Zeit sowie das Familien- und Partnerschaftsklima. Allen TimingGruppen scheint es zu gelingen, eine gute Atmosphäre in der Familie zu schaffen. Dass späte Eltern in dieser Hinsicht besonders erfolgreich wären, kann durch unsere Analyse nicht bestätigt werden (vgl. Hypothese 5). Ebenfalls entgegen den vorliegenden Befunden und entgegen den in Hypothese 5 formulierten Erwartungen nehmen sich späte Eltern nicht mehr Zeit für ihre Kinder als die jüngeren. Sie meinen allerdings besonders häufig, zu viel Zeit für den Beruf zu verwenden. Bei den späten Müttern kann dies mit ihrer im Vergleich zu den jungen und „mittleren“ Müttern sehr viel stärkeren Erwerbseinbindung erklärt werden.

Weitere Unterschiede, die soziale Lagen betreffend, zeigen sich zwischen den Geschlechtern: Vor allem eine frühe Mutterschaft geht mit einer geringen Ressourcenausstattung einher. Viele frühe Mütter bekommen ein erstes Kind, ohne zuvor sicher im Erwerbsleben verankert zu sein. Aber auch mittlere und späte Mütter sind seltener erwerbstätig als Väter der gleichen Timing-Gruppe. Trotz des vergleichsweise hohen Ausbildungsniveaus später Mütter tragen auch bei dieser Timing-Gruppe zumeist die Mütter das Risiko, nach Erwerbsunterbrechungen und Teilzeitphasen beruflich ins Abseits zu geraten. Väter hingegen riskieren, dass sie nach dem Scheitern der Partnerschaft, aus der ihr erstes Kind hervorgegangen ist, mit dem Kind nicht mehr zusammen leben können. Dies erfahren frühe Väter besonders häufig.

Die Befunde machen deutlich, dass frühe Elternschaft häufig mit instabilen Partnerschafts- und Familienformen sowie geringeren ökonomischen und Bildungsressourcen einher geht. Vor diesem Hintergrund erscheint der Trend zum Aufschub der Familiengründung in ein höheres Alter rational. Eine subjektiv befriedigendere Work-Life-Balance und mehr Zeit für ihre Kinder sind allerdings auch späten Eltern nicht vergönnt. Im Gegenteil: späte Eltern sind mit ihrer Zeitverwendung besonders häufig unzufrieden.

\section{Literatur}

Anslinger, E. (2009). Junge Mütter im dualen System der Berufsbildung. Potenziale und Hindernisse. Bielefeld: W. Bertelsmann Verlag (Reihe Berufsbildung, Arbeit und Innovation. Dissertationen/ Habilitationen, Band 16). 
Bundesministerium für Arbeit und Sozialordnung (BMA) (Hrsg.) (2001). Lebenslagen in Deutschland. Der erste Armuts- und Reichtumsbericht der Bundesregierung, Bundestagsdrucksache Nr. 14/5990.

Bundeszentrale für gesundheitliche Aufklärung (BZgA) (2009). Vorwort. In: Bundeszentrale für gesundheitliche Aufklärung (Hrsg.), Schwangerschaft und Schwangerschaftsabbruch bei minderjährigen Frauen. Eine Studie im Auftrag des Bundesverbandes der pro familia, gefördert durch die BZgA. Köln: Bundeszentrale für gesundheitliche Aufklärung, S. 9 (Forschung und Praxis der Sexualaufklärung und Familienplanung).

Block, K., Schmidt, G. (2009). Jugendliche Schwangere und ihre Partner. In: Bundeszentrale für gesundheitliche Aufklärung (Hrsg.), Schwangerschaft und Schwangerschaftsabbruch bei minderjährigen Frauen. Eine Studie im Auftrag des Bundesverbandes der pro familia, gefördert durch die $B Z g A$. Köln: Bundeszentrale für gesundheitliche Aufklärung, S. 41-57 (Forschung und Praxis der Sexualaufklärung und Familienplanung).

Bonell, Chris (2004). Why is teenage pregnancy conceptualized as a social problem? A review of quantitative research from the USA and UK, Culture, Health and Sexuality, 6, 3, S. 255-272.

Cornelißen, W. \& Fox, K. (Hrsg.) (2007). Studieren mit Kind. Die Vereinbarkeit von Studium und Elternschaft: Lebenssituationen, Maßnahmen und Handlungsperspektiven. Wiesbaden: VS Verlag für Sozialwissenschaften (Schriften des Deutschen Jugendinstituts: Gender).

Engstler, H. \& Lüscher, K. (1991). Späte erste Mutterschaft. Ein neues biographisches Muster der Familiengründung? Zeitschrift für Bevölkerungswissenschaft, 17, 4, S. 433-460.

Fosen-Schlichtinger, P. (2006). Späte Mütter. Psychologie heute, 33, 2, S. 62-65.

Friedrich, M. \& Remberg, A. (unter Mitarbeit von Geserick, C.) (2005). Wenn Teenager Eltern werden ... Lebenssituation jugendlicher Schwangerer und Mütter sowie jugendlicher Paare mit Kind. Köln: Bundeszentrale für gesundheitliche Aufklärung (Forschung und Praxis der Sexualaufklärung und Familienplanung).

Friese, M. (2008). Kompetenzentwicklung für junge Mütter. Förderansätze der beruflichen Bildung. Bielefeld: W. Bertelsmann Verlag.

Fthenakis, W. E. et al. (1999). Engagierte Vaterschaft. Die sanfte Revolution in der Familie. Opladen: Leske + Budrich (herausgegeben von der LBS-Initiative Junge Familie).

Grunow, D. (2007). Wandel der Geschlechterrollen und Väterhandeln im Alltag. In: Mühling, T. \& Rost, H. (Hrsg.), Väter im Blickpunkt. Perspektiven der Familienforschung. Opladen: Verlag Barbara Budrich, S. 49-76.

Heinzelmann, C. (2003). Zum Sozialprofil später Mütter. In: Herlyn, I. \& Krüger, D. (Hrsg.), Späte Mütter. Eine empirisch-biographische Untersuchung in West- und Ostdeutschland. Opladen: Leske + Budrich, S. 63-82.

Helfferich, C. Hendel-Kramer, A. \& Wehner, N. (2007). „Irgendwas muss leiden ...“ - Zeit und Zeitplanung studierender Eltern. In: W. Cornelißen, W. \& Fox, K. (Hrsg.), Studieren mit Kind: die Vereinbarkeit von Studium und Elternschaft: Lebenssituationen, Maßnahmen und Handlungsperspektiven. Wiesbaden: VS Verlag für Sozialwissenschaften, S. 51-68 (Schriftenreihe des Deutschen Jugendinstituts: Gender).

Herlyn, I., \& Krüger, D. (2003). Späte Mütter. Eine empirisch-biographische Untersuchung in West- und Ostdeutschland. Opladen: Leske + Budrich.

Hobcraft, J. (2008). The timing and partnership context of becoming a parent: Cohort and gender commonalities and differences in childhood antecedents. Demographic Research, 19, S. 12811322.

Institut für Demoskopie Allensbach (2004). Einflussfaktoren auf die Geburtenrate. Ergebnisse einer Repräsentativbefragung der 18- bis 44-jährigen Bevölkerung. http://www.ifd-allensbach.de/pdf/akt_ 0407.pdf [Stand; 2007-09-26].

Jurczyk, K., Keddi, B., Lange, A. \& Zerle, C. (2009). Zur Herstellung von Familie. Wie sich die Alltagspraxis von Vätern, Müttern und Kindern erforschen lässt - ein Werkstattbericht. DJI Bulletin, 82, Bulletin Plus.

Jürgens, K. (2001). Familiale Lebensführung. In: Voß, G. \& Weihrich, M. (Hrsg.), Tagaus, tagein. Neue Beiträge zur Soziologie alltäglicher Lebensführung. München/Mehring: Rainer Hampp Verlag, S, 33-60. 
Klindworth, H., Walter, W. \& Helfferich, C. (2004). Frühe erste Vaterschaft. Ein intendierter, passender Übergang? In: Tölke A. \& Hank, K. (Hrsg,), Männer - Das ,,vernachlässigte " Geschlecht in der Familienforschung. Wiesbaden: VS Verlag für Sozialwissenschaften, S. 152-177 (Sonderheft 4 der Zeitschrift für Familienforschung).

Marsiglo, W. \& Cohan, M. (1997). Young fathers and child development. In: Lamb, M. E. (Hrsg.), The role of the father in child development. New York: John Wiley \& Sons, S. 227-244.

Matthiesen, S., Schmidt, G. (2009). Ausgang der Schwangerschaft. In: Bundeszentrale für gesundheitliche Aufklärung (Hrsg.), Schwangerschaft und Schwangerschaftsabbruch bei minderjährigen Frauen. Eine Studie im Auftrag des Bundesverbandes der pro familia, gefördert durch die BZgA. Köln: Bundeszentrale für gesundheitliche Aufklärung, S. 59-68 (Forschung und Praxis der Sexualaufklärung und Familienplanung).

Middendorff, E. (2008). Studieren mit Kind. Ergebnisse der 18. Sozialerhebung des Deutschen Studentenwerks durchgeführt durch HIS Hochschul-Informations-System. Berlin: Bundesministerium für Bildung und Forschung (Hrsg,) http://www.stuki-hu.de/docs/18sozialerhebung0308.pdf [Stand: 2011-01-25].

Mühling, T. (2005). Lebenslagen von Niedrigeinkommenshaushalten - eine Analyse des NIEP. Zeitschrift für Familienforschung, 17, 2, S. 167-188.

Payk, B. \& Schmider, A. (2009). Kinder? Jetzt nicht! - Aber wann dann? Wie Arbeitsplatzunsicherheit die Familiengründung beeinträchtigt. Statistisches Monatsheft Baden-Württemberg, 8, S. 11-14.

Scharein, M. G. (2010). Kommen die Kinder immer später, so verzerrt das die Statistik... - Zur Schätzung und Interpretation der durchschnittlichen Kinderzahl je Frau. Bevölkerungsforschung Aktuell, 4, S. 22-23.

Schmidt, G. (2009). Jugendsexualität und Jugendschwangerschaften: Zeitliche Trends. In: Bundeszentrale für gesundheitliche Aufklärung (Hrsg.), Schwangerschaft und Schwangerschaftsabbruch bei minderjährigen Frauen. Eine Studie im Auftrag des Bundesverbandes der pro familia, gefördert durch die BZgA. Köln: Bundeszentrale für gesundheitliche Aufklärung, S. 13-27 (Forschung und Praxis der Sexualaufklärung und Familienplanung).

Schulz, F. \& Blossfeld, H.-P. (2006). Wie verändert sich die häusliche Arbeitsteilung im Eheverlauf? Eine Längsschnittstudie der ersten 14 Ehejahre in Westdeutschland. Kölner Zeitschrift für Soziologie und Sozialpsychologie, 58, 1, 23-49.

Spies, A. (2009). Frühe Mutterschaft. Junge Frauen im Klammergriff von Normen, Mythen und Struktur. Sozial Extra, 33, 5/6, S. 15-19.

Spies, A. (2010). Frühe Mutterschaft - Eine pädagogische Herausforderung der besonderen Art. Einleitung. In: Spies, A. (Hrsg.), Frühe Mutterschaft. Die Bandbreite der Perspektiven und Aufgaben angesichts einer ungewöhnlichen Lebenssituation, Baltmannsweiler: Schneider Verlag Hohengehren, S. 9-24.

Statistisches Bundesamt (2003). Statistik der Geburten. Wiesbaden: Statistisches Bundesamt.

Statistisches Bundesamt \& Bundesministerium für Familie, Senioren, Frauen und Jugend (Hrsg.) (2003). Wo bleibt die Zeit? Die Zeitverwendung der Bevölkerung in Deutschland 2001/02. Wiesbaden: Statistisches Bundesamt.

Statistisches Bundesamt (2007). Geburten in Deutschland. Wiesbaden: Statistisches Bundesamt. http://www. destatis.de/jetspeed/portal/cms/Sites/destatis/Internet/DE/Content/Publikationen/Fachveroeffentlichungen/ Bevoelkerung/BroschuereGeburtenDeutschland,property=file.pdf [Stand: 2010-10-31].

Statistisches Bundesamt (2010). Pressemeldung Nr. 414 des Statistischen Bundesamtes vom 12.11.2010. Wiesbaden: Statistisches Bundesamt.

Stauber, B. (2010): Unter widrigen Umständen - Entscheidungsfindungsprozesse junger Frauen und Männer im Hinblick auf Familiengründung. In: Spies, A. (Hrsg.), Frühe Mutterschaft. Die Bandbreite der Perspektiven und Aufgaben angesichts einer ungewöhnlichen Lebenssituation, Baltmannsweiler: Schneider Verlag Hohengehren.

Yarrow, A. L. (1991). Latecomers. Children of parents over 35. New York: The Free Press.

Zerle, C. \& Krok, I. (2008). Null Bock auf Familie? Der schwierige Weg junger Männer in die Vaterschaft. Gütersloh: Verlag Bertelsmann Stiftung (Bertelsmann Stiftung, Hrsg,). 
Eingereicht am/Submitted on: 27.05.2011

Angenommen am/Accepted on: 14.09.2011

Anschrift der Autorinnen und des Autors/Addresses of the authors:

Claudia Zerle-Elsäßer, Diplom-Soziologin

PD Dr. Waltraud Cornelißen

Dr. Walter Bien

Deutsches Jugendinstitut (DJI)

Nockherstraße 2

81541 München

Deutschland/Germany

E-Mail: zerle@dji.de cornelissen@dji.de bien@dji.de

\section{Anhang}

Tabelle A1: Multinomial logistische Regression, Mütter, Referenzkategorie=späte Mütter

\begin{tabular}{|c|c|c|}
\hline \multirow[t]{2}{*}{ Prädiktoren } & \multicolumn{2}{|c|}{ Odds Ratios } \\
\hline & Frühe Mütter & Mittlere Mütter \\
\hline \multicolumn{3}{|l|}{ Höchster Schulabschluss } \\
\hline Hauptschulabschluss (1) & 2,26 & 0,79 \\
\hline \multicolumn{3}{|l|}{ Mittlere Reife/ } \\
\hline Realschulabschluss (2) & $3,92^{* * *}$ & $1,91^{* *}$ \\
\hline Fachhochschulreife (3) & 1,65 & 1,27 \\
\hline Abitur (0) & . & . \\
\hline \multicolumn{3}{|l|}{ Persönliches Nettoeinkommen } \\
\hline Kein eigenes Einkommen (1) & $18,22^{* * *}$ & $4,35^{\star * *}$ \\
\hline Unter 999 Euro (2) & $7,30^{* *}$ & $1,96^{*}$ \\
\hline 1000 bis $1999(3)$ & 3,30 & 1,51 \\
\hline 2000 und mehr (0) & . & . \\
\hline \multicolumn{3}{|l|}{ Nettohaushaltseinkommen } \\
\hline Unter 1999 Euro (1) & $3,34^{* *}$ & $2,67^{* *}$ \\
\hline 2000 bis $3999(2)$ & 1,93 & $2,16^{* * *}$ \\
\hline 4000 Euro und mehr $(0)$ & . & . \\
\hline \multicolumn{3}{|c|}{ Noch mit dem Vater des Kindes zusammen } \\
\hline $\mathrm{Ja}(1)$ & $0,18^{* * *}$ & 0,93 \\
\hline Nein $(0)$ & . & . \\
\hline
\end{tabular}

${ }^{*} \mathrm{p}<0.1, * * \mathrm{p}<0.05 * * * \mathrm{p}<0.01$. Das Gesamtmodell ist signifikant $(0.000)$. Die Modellaufklärung ist moderat (Nagelkerkes $\mathrm{R}^{2}=0,198$ ). $\mathrm{N}=650$ (davon 115 frühe Mütter, 427 mittlere Mütter und 108 späte Mütter)

Quelle: AID:A - DJI-Survey 2009; 18- bis 55-Jährige mit leiblichen Kindern unter 7 Jahren; N=1.239 
Tabelle A2: Multinomial logistische Regression, Väter, Referenzkategorie späte Väter

\begin{tabular}{|c|c|c|}
\hline \multirow[t]{2}{*}{ Prädiktoren } & \multicolumn{2}{|c|}{ Odds Ratios } \\
\hline & Frühe Väter & Mittlere Väter \\
\hline \multicolumn{3}{|l|}{ Höchster Schulabschluss } \\
\hline Hauptschulabschluss $(1)^{28}$ & 1,78 & 0,52 \\
\hline \multicolumn{3}{|l|}{ Mittlere Reife/ } \\
\hline Realschulabschluss (2) & $2,71^{\star \star *}$ & 1,02 \\
\hline Fachhochschulreife (3) & 1,99 & 0,76 \\
\hline Abitur (0) & & . \\
\hline \multicolumn{3}{|l|}{ Persönliches Nettoeinkommen } \\
\hline Unter 999 Euro (1) & $12,41^{* * *}$ & 1,36 \\
\hline 1000 bis $1999(2)$ & $2,31^{* *}$ & 1,07 \\
\hline 2000 und mehr $(0)$ & . & . \\
\hline \multicolumn{3}{|l|}{ Nettohaushaltseinkommen } \\
\hline Unter 1999 Euro (1) & $4,29^{* *}$ & $3,34^{*}$ \\
\hline 2000 bis 3999 (2) & $3,81^{* * *}$ & $2,20^{\star \star *}$ \\
\hline 4000 Euro und mehr (0) & & . \\
\hline \multicolumn{3}{|c|}{ Noch mit der Mutter des Kindes zusammen } \\
\hline $\mathrm{Ja}(1)$ & $0,08^{\star *}$ & 0,95 \\
\hline Nein $(0)$ & & \\
\hline
\end{tabular}

$*_{\mathrm{p}}<0.1, * * \mathrm{p}<0.05 * * * \mathrm{p}<0.01$. Das Gesamtmodell ist signifikant $(0.000)$. Die Modellaufklärung ist in Ordnung (Nagelkerkes $\mathrm{R}^{2}=0,302$ ).

Quelle: AID:A - DJI-Survey 2009; 18- bis 55-Jährige mit leiblichen Kindern unter 7 Jahren; N=1.239

28 Die Interpretation der multinomial logistischen Regression ist etwas kompliziert, weil bei kategorialen unabhängigen Variablen zwei Referenzkategorien vorliegen, also eine Kategorie der abhängigen Variable und eine Kategorie der unabhängigen Variable (im Modell durch 0 gekennzeichnet). Die Interpretation erfolgt folgendermaßen: Die Chance, eher den frühen als den späten Müttern anzugehören, ist bei einer Person mit Hauptschulabschluss, gegenüber einer Person mit Abitur, 7,45 fach größer. 